\title{
A NEW TYPE OF BETATRON WITHOUT AN IRON YOKE
}

\author{
By A. BIERMAN \\ Philips Research Laboratories, Eindhoven
}

\begin{abstract}
RY far the most expensive and the heaviest part of betatrons, as usually constructed, is the iron yoke with its complicated pole-pieces. We propose to describe a mode of construction which uses no yoke, and which, though restricted in its applications, offers several important advantages over the original type of betatron.
\end{abstract}

The main features of our betatron are two coils in series, with a sealed-off glass acceleration tube between them. The dimensions are chosen so as to fulfil the well-known condition $0<n<1, n$ being determined by the radial decrease of the magnetic field, which is assumed to be proportional to $r-n$. The flux required within the electron orbit is attained by means of a small iron core placed in the axis of the coils. This iron core is inserted inside the 'Pertinax' tube, visible in the photograph. Saturation of this core effects contraction of the orbits towards the end of an acceleration period, until the electrons strike a tungsten target $0.1 \mathrm{~mm}$. thick. The core, made of transformer sheet-iron, is interrupted half-way by a small gap which is so adjusted that the contribution of its stray field makes $n=\frac{1}{2}$ over almost the entire area of the electron orbits.

The dimensions of the tube are hence not restricted by pole pieces, and have been made very large. The inner wall of the tube is covered with a transparent, fluorescent semi-conductor, which has proved valuable for investigating the behaviour of the electron beam at different magnetic field-strengths and the effects of parasitic fields. Moreover, the transparency of the tube is very convenient for the glass-blower, while the electron gun and the target are being sealed into their precise positions. The electron gun is small both in the axial and radial direction; hence its chance of being struck by electrons is less than with the usual type of con. struction.

If simplicity of equipment is desired, we may forgo the advantage of short-pulse injection and take a sinusoidal injection voltage from an auxiliary coil placed on the axis of the main coils (shown on the left of the photograph). The tension induced in this coil amounts to several kilovolts, from which the injection voltage can be tapped off at various points.

The current through the coils is supplied by discharging a $6 \cdot 5 \mu \mathrm{F}$. condenser via a spark gap. The condenser is continuously loaded from a rectifier, and the spark gap breaks down as soon as the voltage across the condenser reaches a value of about $50 \mathrm{kV}$., so that the discharges are automatically repeated at intervals of a few seconds. It is also possible to arrange for a single discharge at a fixed moment, as is, for example, required for Wilson camera exposures.
Each discharge initiates damped oscillations with a frequency of $2,500 \mathrm{c} . / \mathrm{sec}$, in which the peak current through the coils attains a value of about 5,000 A. The power needed to maintain undamped oscillations would, with the present construction, be several thousand kilowatts, which makes continuous operation impossible because the dissipation would then be far too high. The small apparatus, shown in the photograph, is therefore only capable of producing radiation pulses at intervals of at least one second. Since after a few complete oscillations the current has been so much reduced that the iron core is no longer sufficiently saturated, only a few periods can be utilized in each discharge.

Though the mechanical forces resulting from the very heavy currents demand a solid construction of the coil frame, the betatron as a whole weighs no more than $50 \mathrm{kgm}$., the iron core itself weighing less than $5 \mathrm{kgm}$.

As the peak value of the magnetic field is 0.4 V.sec. $/ \mathrm{m}^{2}{ }^{2}(4,000$ gauss) and the radius of the orbit is $8 \mathrm{~cm}$., the energy of the accelerated electrons amounts to $9 \mathrm{MeV}$. This value can be reduced by exchanging the core for a smaller one which is saturated at lower currents ; the contraction of the electron orbits will then take place at an earlier phase of the discharge. In this case a greater number of periods are utilized in each discharge.

It will be clear that in experiments requiring shortwave X-ray output during a brief period (as, for example, nuclear processes investigated with a Wilson camera) this type of betatron offers distinct advantages. Its simple construction makes the cost low, and it is light enough to be comparatively easy to handle ;

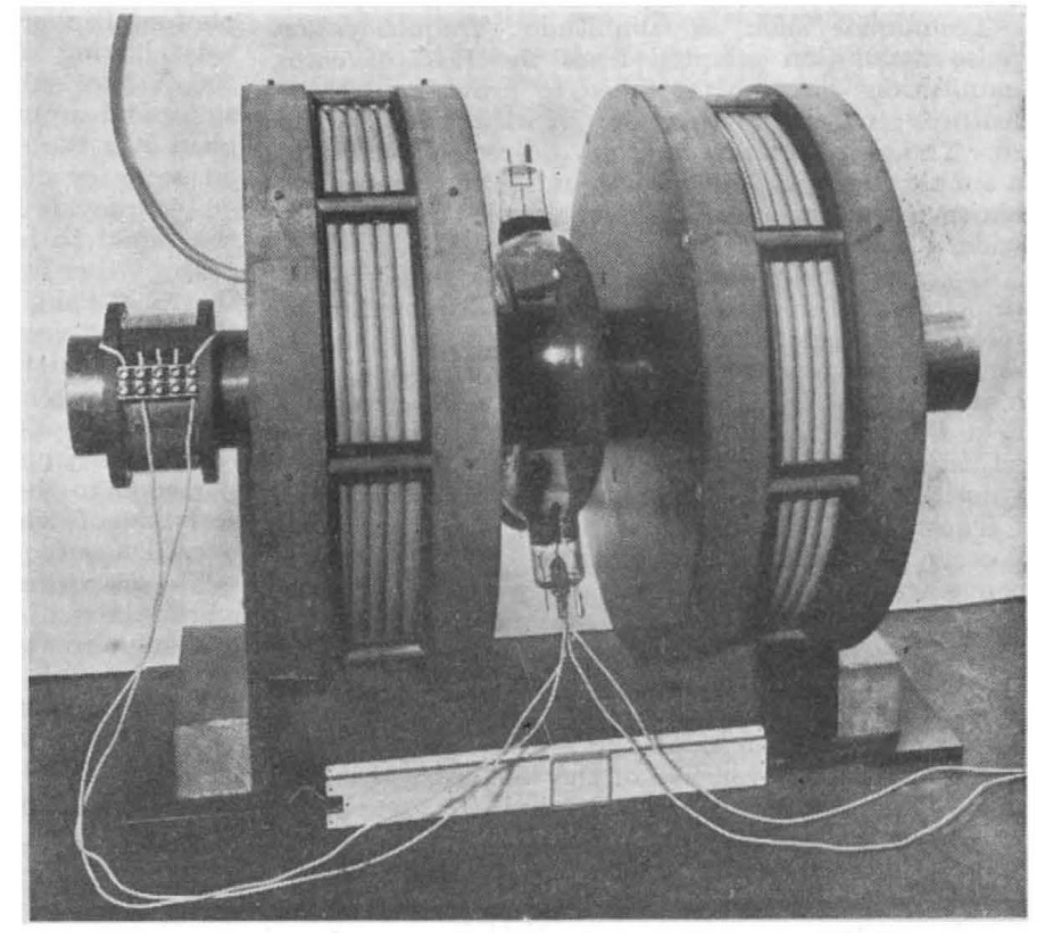

9-MeV. betatron without an iron yoke. Slide-rule against base gives the scale 
it can, for example, be mounted on a mobile support. Moreover, the intensity of the radiation produced is greater than with the customary betatrons, for several reasons : because of the high frequency, the energygain in each loop is considerable (240 volts); this effects strong damping of the oscillations about the equilibrium orbit, while, moreover, the total path of the electrons is not more than $30 \mathrm{~km}$. Consequently the loss of electrons by impact against the wall or against the molecules of the residual gas is considerably smaller than at the lower frequencies commonly used. In addition, a greater number of electrons can be accelerated at a time, owing to the large dimensions of the acceleration tube. Lastly, the axial symmetry of the magnetic field is more perfect than in an apparatus with pole pieces arranged in sectors, which again reduces the loss of electrons. Reliable data on the intensity of the radiation are not as yet available, but our experiments have already yielded evidence that the peak intensity is much higher than that obtained, in this laboratory, with a betatron of the customary type.

\section{MULTIPLICATION AND DIVISION BY ELECTRONIC-ANALOGUE METHODS}

\author{
By E. M. DEELEY and D. M. MACKAY \\ Wheatstone Laboratory, King's College, London
}

$\mathrm{T}$ HE product or quotient of two rapidly varying quantities is difficult to obtain by conventional electronie-analogue methods. Desirable features in a multiptier include: $(a)$ symmetry of response to positive and negative inputs; $(b)$ an absolute indication of zero input, with consequent freedom from zero drift; $(c)$ independence of normal changes in electponic characteristics ; $(d)$ rapidity and accuracy of response.

Techniques such as amplitude-, frequency- or pulse-modulation adapted from the field of communications have so far failed to provide all these features ; most are fundamentally incapable of doing so. The object of this note is to direct attention to a simple principle which has been found to meet the above requirements ${ }^{1}$, and which seems to invite wider application in this and related fields.

It is most easily described as a development of the principle used in J. J. Thomson's familiar method of measuring electron velocity, in which the force on an electron moving with velocity $v$ at right-angles to a magnetic field $H$ is counteracted by an electrostatic field $E$ proportional to the product $H \times v$. If the adjustment of $E$ were automatic and instantaneous, its value would be a continuous measure of this product.

The cathode-ray tube, which is well known to possess potentially all four virtues above, forms a convenient basis for experimental work. In Thomson's arrangement, however, $v$ was the axial velocity of the electron stream, which is not a widely variable or reversible quantity. Accordingly, in the practical form of multiplier shown in essentials in the accom. panying diagram, an axial magnetic field is used and $v$ is the transverse velocity of the electron stream in the $X$-direction, proportional to a voltage $V_{x}$ applied to conventional deflecting plates $X_{1}, X_{2}$. After $X$-deflexion, the stream passes through the magnetic field $H$, which produces a deflecting force, initially in the $Y$-direction, proportional to $H \times V_{x}$.

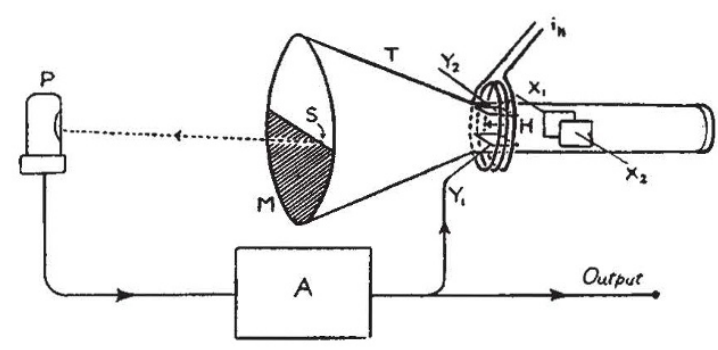

The $Y$-deflexion of the spot $S$ on the screen, which would normally result (corresponding to a rotation of the pattern), is counteracted by a photo-electric feedback system of the type used in function. generators ${ }^{2,3}$. The photocell $P$, coupled to one of the $Y$-plates via the amplifier $A$, prevents the spot from deviating by more than a fraction of its diameter from the edge of the mask $M$. With care in adjust. ment of the latter, the spot can thus be held closely to the $X$-axis, and $V_{y}$ is then proportional to the product of $V_{x}$ with the current $i_{H}$ producing $H$. Conversely, if the photocell is used to control $H$ and voltages $V_{x}, V_{y}$ are applied to $X$ and $Y$ plates, the current $i_{H}$ is proportional to the quotient $V_{y} / V_{x}$.

Since the important parameters other than the accelerating voltage are purely geometrical, the prospects of attaining high stability in a device of this kind are good. Output is sensibly independent of changes in the gain of $A$, as usual in feedback circuits. At a later stage, it is hoped to dispense with the photo-electric link, using a collecting. electrode inside the tube to perform the combined function of cell and mask.

At the time of writing, it has not been possible to make a fair test of the accuracy and frequency-range of the technique; but it may be of interest to sum. marize the encouraging results of preliminary experiments with quite crude apparatus. A 931-A photomultiplier has been used, with a cathode-ray tube having a blue screen and running at only $500 \mathrm{~V}$. to enhance overall sensitivity; D.c. and sinusoidal inputs up to $10 \mathrm{kc}$. $/ \mathrm{sec}$. have been multiplied over the ranges $\pm 300 \mathrm{~mA}$. and $\pm 20 \mathrm{~V}$., with an accuracy of the order of 2 per cent at $50 \mathrm{c} . / \mathrm{sec}$.

Accuracy is limited in practice fundamentally by the signal to noise ratio attainable in the feedback path. Other important factors are the uniformity of the axial field, and its effective axial length, which should not greatly exceed that of the $Y$-plates. If magnetic $X$-deflexion were used, the $Y$-voltage would be practically independent of 'gun' voltage; but if electrostatic $X$-plates are employed for the sake of their high impedance, the output is directly proportional to the square root of the gun voltage, the stability of which is then an important factor in overall accuracy.

The importance of such devices to the physicist and mathematician lies chiefly in their application to the solution by analogue methods of differential equations with non-constant coefficients. A number of further applications of the same principle to highspeed electronic computing technique are being developed, and a fuller account of these and the above will be published elsewhere.

\footnotetext{
${ }^{1}$ MacKay, D. M., Brit. Prov. Pat. Spec. No. 11161 (1948). The same principle has been the subject of an unpublished independent investigation by D. J. Mynall.

${ }^{2}$ MacKay, D. M., Nature, 159, 406 (1946).
}

${ }^{3}$ Mynall, D. J., Nature, 159, 743 (1946). 